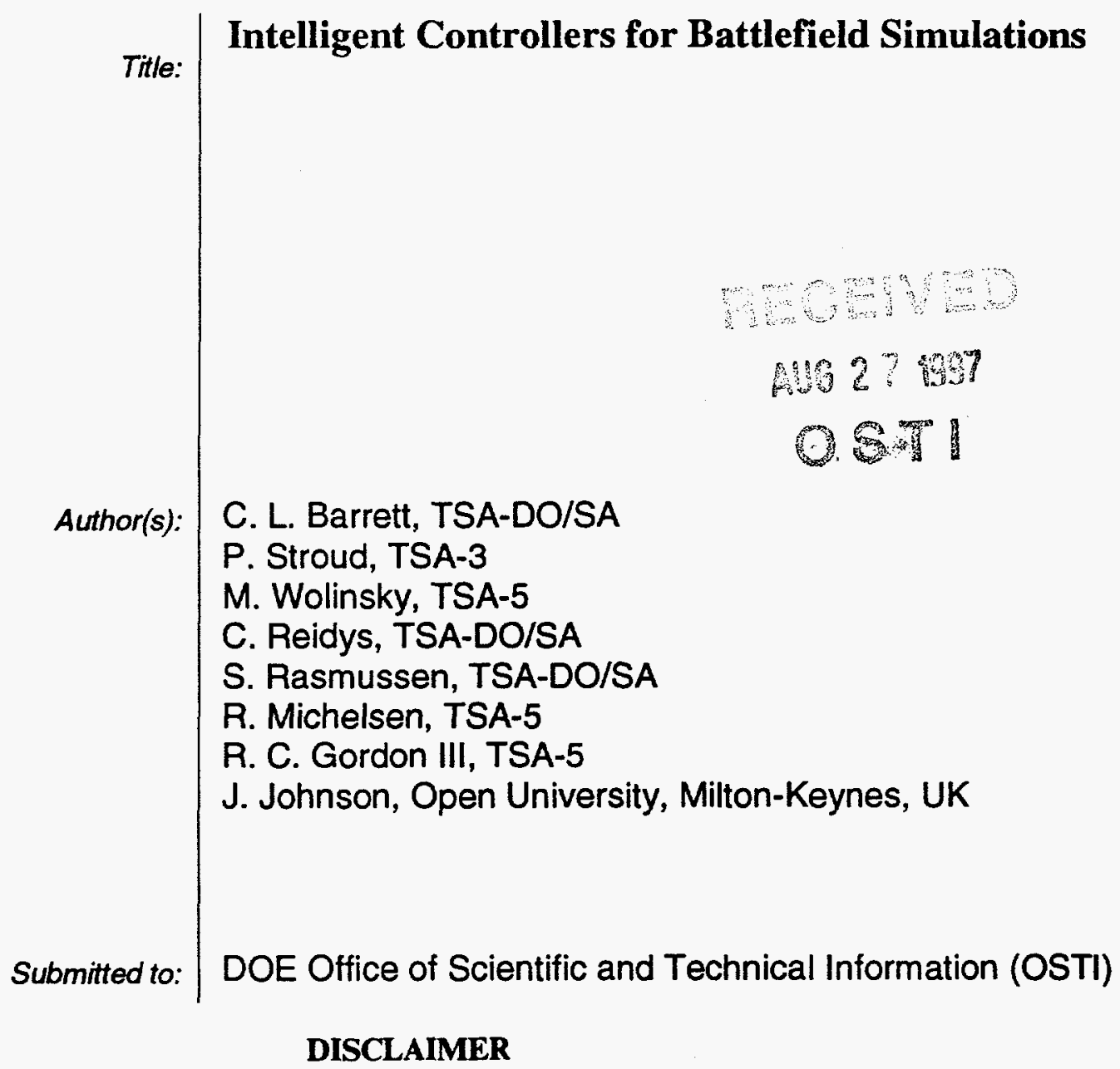

\begin{abstract}
This report was prepared as an account of work sponsored by an agency of the United States Government. Neither the United States Government nor any agency thereof, nor any of their employees, makes any warranty, express or implied, or assumes any legal liability or responsibility for the accuracy, completeness, or usefulness of any information, apparatus, product, or process disclosed, or represents that its use would not infringe privately owned rights. Reference herein to any specific commercial product, process, or service by trade name, trademark, manufacturer, or otherwise does not necessarily constitute or imply its endorsement, recommendation, or favoring by the United States Government or any agency thereof. The views and opinions of authors expressed herein do not necessarily state or reflect those of the United States Government or any agency thereof.
\end{abstract}

Los Alamos National Laboratory, an altimative action/equal opportunity employer, is operaled by the University of California for the U.S. Department of Energy under contract W-7405-ENG-36. By acceptance of this article, the publisher recognizes that the U.S. Government retains a nonexclusive, royallyIree license to publish or reproduce the published form of this contribution, or to allow others to do so, for U.S. Government purposes. Los Alamos National Laboratory requests that the publisher identify this article as work periormed under the auspices of the U.S. Department of Energy. Los Alamos Nallonal Laboratory strongly supports academic treedom and a researcher's right to publish; as an institution, however, the Laboratory does not endorse the vieupoint of a publication or guarantee its tectnical correctness. 


\section{DISCLAMIER}

Portions of this document may be illegible in electronic image products. Images are produced from the best available original document. 


\title{
Intelligent Controllers for Battlefield Simulations
}

\author{
Christopher L. Barrett,* Phillip Stroud, Murray Wolinsky, Christian Reidys, \\ Steen Rasmussen, Randy Michelsen, and Ray C. Gordon III \\ Los Alamos National Laboratory \\ Jeffrey Johnson \\ Open University, Milton-Keynes, UK
}

\begin{abstract}
This is the final report of a three-year, Laboratory-Directed Research and Development (LDRD) project at the Los Alamos National Laboratory (LANL). This project involved research in the area of warfighting simulation technology and methods. In the first year our emphasis was to produce a prototype repository for simulation software objects and simulation execution executives in the context of an integrated theater missile defense problem. This provided a necessary precursor to the detailed development of more comprehensive simulation software composition environments. In the second year more detailed and complete development of composable simulation software was undertaken. An object description language was developed, an object composition architecture was developed and implemented, and a prototyping test bed was produced to assess the technical concepts and as a demonstration tool for programmatic activities. In the third and final year it became apparent that the representation of intelligent entities, specifically those that fulfill command and control functions in warfighting systems, was a challenging and serious technical shortcoming of all existing approaches to simulation of warfare.
\end{abstract}

\section{Background and Research Objectives}

The nation's leadership recognized that a new defense strategy is necessary to respond to a dramatically altered and more dynamic security environment. Focus is shifting from forward basing and theater level combat against a sophisticated, well understood threat to a broader, more diverse mission spectrum ranging from humanitarian efforts through low intensity conflicts (such as counter-drug operations) to power projection resulting in smaller scale and potentially multiple, simultaneous tactical level engagements. Significantly, these changes occur during a time of declining resources. Consequently a new defense acquisition strategy is evolving that attempts to respond to

*Principal Investigator, E-mail: barrett@tsasa.lanl.gov 
these new challenges while recognizing fiscal realities. The old strategy that places a premium on rapid development and procurement of new technologies to counter Soviet advances had given way to a more circumspect and deliberate approach that emphasizes longer periods for research, development, testing, and proving the military value of technologies before procurement. This requirement to more rigorously demonstrate technical feasibility, affordability, and operational utility "up-front" will result in an unprecedented use of, indeed reliance on, computer models and simulations. At this critical juncture, the existing generation of military models and simulations, developed and optimized over decades to analyze the Warsaw Pact threat, is simply not capable of providing accurate nor credible analysis of current and future combat environments.

This research was designed to develop a synthetic battlefield environment that combines the analytic rigor of high resolution combat models and simulations with the intuitively appealing virtual reality of man-in-the-loop simulators when desired. The intent was to complement current Department of Defense (DoD) efforts as proof-ofprincipal.

\section{Importance to LANL's Science and Technology Base and National R\&D Needs}

Most broadly, this effort attempted to establish the Laboratory in a position of leadership in defense technology research, development, testing, and evaluation where our unique capabilities match DoD needs. This project directly supports many of the implementation strategies in the Defense Technologies subsector of the Laboratory Strategic Plan. Primary among them are:

- Support the development of architectures for synthetic environment and virtual reality for the defense infrastructure of the future.

- Focus system simulation activities to directly impact science and technology strategies for the development and evaluation of future DoD requirements.

- Develop closer relationships and strategic alliances with the services and the service laboratories in support of science and technology and advanced technology demonstrations (ATDs).

- Support the Director of Defense Research and Engineering (DDR\&E) Synthetic Environments Thrust Area. 
- Develop the state of the art in simulated command and control (C2) decision making in intelligent software actors.

\section{Scientific Approach and Accomplishments}

In the first year the project emphasis was to produce a prototype repository for simulation software objects and simulation execution executives in the context of an integrated theater missile defense problem. This provided a necessary precursor to the detailed development of more comprehensive simulation software composition environments. In the second year more detailed and complete development of composable simulation software was undertaken. An object description language was developed, an object composition architecture was developed and implemented, and a prototyping test bed was produced to assess the technical concepts and as a demonstration tool for programmatic activities. In the third and final year it became apparent that the representation of intelligent entities, specifically those that fulfill command and control functions in warfighting systems, was a challenging and serious technical shortcoming of all existing approaches to simulation of warfare.

Because of the programmatic opportunities offered by the intelligent actor representation problem in large DoD simulation initiatives and the special interest in that area posed by international forces, joint forces, and the realities of low-intensity conflict and peace-keeping missions as well as US joint forces restructuring, we undertook a serious effort in the third year to develop the state of the art in simulated C2 decision making in intelligent software actors. Specifically in the third year the emphasis was planned to be in five areas:

- direct acquisition of logical rules for agent control using adaptive methods,

- modification of rules by simulated experience,

- minimal object complexity,

- intelligent control architecture for command, control, communications, and intelligence (C3I): representing echelon, and

- memory utilization of adaptive representations

Methods were developed for acquisition of logical control behaviors using multilinear fuzzy networks and genetic algorithms. These were demonstrated in simple tank-target-acquisition logic and in more complex airborne-laser fire-control settings. The methods are new, robust, and clearly demonstrate the viability of the approach. In simulated experience-based learning, the airborne laser setting clearly demonstrated the 
potential of the rule representations to evolve to improved performance levels, thereby essentially producing a modest amount of original tactical logic. This effort clearly demonstrated that in actual practice we can develop complex control logic that both incorporates existing knowledge (e.g., tactics and doctrine) and provides for innovation and discovery.

However, several important issues must be addressed prior to employment of these techniques in the composition environments provided during the first two years of research. First, the impact of local rules in interaction is a very deep and difficult problem. A consequence of this is that it is difficult to know when the encoded rules are minimal in any sense. That is, when are we both encoding and storing as little as possible to produce valid entity behaviors and when might we be accidentally over-prescribing behavior.

Initially research focused on a particular simulation-based approach looking at hierarchies in molecular self-assembly. It seemed to be a good starting point for this question, but it became clear that because of conceptual defects in the approach and the inability to transfer results easily to more cognitive/decision process settings, we required a different approach.

We assessed the impact of evolution of the learning systems in the first task on the dynamically composed system. Using a setting that employed simulated drivers on a roadway, we determined that it is possible to observe adaptive behavior among interacting simulated actors without internal learning mechanisms. This led us to very seriously undertake a mathematical analysis of the basic mechanism of simulated interactions. This mechanism is the simulation state update, or logically consistent iteration of the interacting dynamical entities. We found that this is a very rich mathematical area that, in addition to giving us the basis to rigorously address the issue of minimal object representation, is indeed related to molecular structure. What we found is essentially that the inter-actor causal relations that continuously vary as the simulation unfolds are a rich source of structure in the observed system behavior. This entirely new result has an amazing and complete mathematical metaphor in the constraints imposed by Watson-Crick pairs in the bimolecular structure of RNA. We have been able to exploit this mathematics to begin to comprehend what can be rendered structurally invariant in an entity representation and thereby reduce to an absolute minimum the state transition calculations required to produce a given simulated behavior. Thus, we would minimize both static and dynamic memory requirements as well as increasing runtime speed of the calculations required to produce the actor interactions. 
However, interpreting the "intelligence" due to local rules and that aspect of apparent intelligence due to the effects of interactions became more difficult. This imposes design as well as analysis challenges to the practitioner. We employed concepts from the KOALAS intelligent control architecture developed several years ago in related LDRD research in the area of intelligent control to attempt to sort out this problem. The design architecture it provides can also be used to serve an analysis role and allow us to interpret emergent functions that are produced by the interactions of local rules in the context of the functional requisites of intelligent control. We established that the architecture had relevance to the ground, air, and sea combat C2 setting. Moreover the architecture naturally addresses concerns in the professional military community about the viability of attempting to use logical methods to represent every aspect of the $C 2$ process. The architecture allows a separation of interpretive and inductive functions from deductive reasoning. Thus we have a framework within which we can offer the prospects for interpretation of the minimal actor representations as intelligent $\mathrm{C} 2$ entities.

Moreover, the issue of echelon in C4I, which is the embodiment of hierarchical phenomena in warfighting systems, has a natural representation in the emergent phenomena produced by interacting local rules. We have undertaken, but have not completed a study wherein very simple and local rules pertaining to field of fire boundaries induce verifiable unit $\mathrm{C} 2$ and tactics in a simulation without explicit encoding of the unit tactics.

The timeliness and importance of the technical results of this project have been reflected in programmatic successes. The composition environments and composed entity methods have resulted in adoption of the Los Alamos warfighting simulation project, JOINTSIM, as the research platform for major simulation programs in the Office of the Secretary of Defense (OSD) and in the services. This has already resulted in significant funding and important follow-on work for the Army, Air Force, DoD, OSD and others. In addition the canonical $\mathrm{C} 2$ actor architecture, as well as associated concepts developed in the final year of this LDRD project, have been adopted by the joint US/UK effort in warfighting simulation technology and methodology. The joint effort is led in the US by the National Simulation Center at Fort Leavenworth, by the Defence Research Establishment in the UK, and is controlled by the Deputy Undersecretary of the Army for Operations Research. We anticipate many more important opportunities for real impacts of further research in this area. 


\section{Publications}

1. Barrett C., Wolinsky M., and Olesen, M., Emergent Local Control Properties in Particle Hopping Traffic Simulations, Los Alamos National Laboratory report LAUR:95-4368, 1996.

2. Nagel K., Barrett, C., and Rickert, M., Parallel Traffic Microsimulation of Traffic by Cellular Automata and Application for Large-Scale Modeling, Los Alamos National Laboratory report LA-UR:96-0050, 1996.

3. Rasmussen, S., Barrett, C., and Olesen M., Dynamical Hierarchies: A Summary, Los Alamos National Laboratory report LA-UR-96-660, 1996.

4. Reidys, C., Mappings in Random Structures, Los Alamos National Laboratory report LA-UR:96-1641, 1996.

5. Barrett, C. and Reidys, C., Formal Simulation: I. Updates, Los Alamos National Laboratory report (in process).

6. Barrett, C., Johnson J., and Reidys C., Updating Procedural Representations of Dynamical Systems, Los Alamos National Laboratory report (in process).

7. Barrett, C., Jones, R., and Hand U., Adaptive Capture of Expert Knowledge, Los Alamos National Laboratory report LA-UR-95-1391, 1996.

8. Stroud, Phillip, Simulation-Based Learning in Knowledge-Based Controllers, proc. 1996 Int'1 Symposium on Intelligent Controllers, 1996.

9. Stroud, Phillip, Intelligent Actors in Synthetic Environments, Los Alamos National Laboratory report LA-UR-96-131, 1996.

10. Stroud, Phillip and Turner, Bobby, Neural Network Based Training Methods for an Airborne Laser Adaptive Fire Controller, Los Alamos National Laboratory report LA-UR-95-1442, 1996. 